Ann. Biol. anim. Bioch. Biophys., I974, 14 (3), 487-498.

\title{
RÉGULATION \\ DE LA SÉCRÉTION PANCRÉATIQUE PAR RÉTRO-ACTION NÉGATIVE CHEZ LE PORC
}

\author{
T. CORRING \\ avec la collaboration technique de Anne-Marie Guevgneau et C. Guerin \\ Laboratoire de Physiologie de la nutrition \\ Centre national de Recherches zootechniques, I. N. R. A., \\ 78350 Jouy en Josas
}

\section{RÉSUMÉ}

Sur des porcs munis de fistules pancréatique et duodénale, et dont on assure en permanence la réintroduction du suc pancréatique, l'arrêt de cette opération se traduit dans l'heure qui suit par une augmentation de la quantité de protéines totales $(\times \mathrm{I}, 5-2)$ et du volume du suc recueilli $(\times 5)$. Dès que l'on retourne le suc pancréatique à l'animal au niveau duodénal, la sécrétion retrouve sa valeur de base.

Un mélange de sels $\left(\mathrm{NaHCO}_{3}+\mathrm{NaCl}\right)$ représentatif de la composition en ces mêmes sels du suc pancréatique, ou un suc pancréatique déprotéinisé n'ont aucun effet inhibiteur sur la sécrétion du pancréas lorsqu'ils sont perfusés dans le duodénum.

Par ailleurs l'introduction intraduodénale d'une solution de trypsine active, même à pH acide, conduit à une diminution de la sécrétion pancréatique, identique à celle induite par le suc pancréatique réintroduit.

Il semble donc que seules les enzymes et plus précisément les enzymes protéolytiques soient à l'origine de ce phénomène de rétro-action négative de la sécrétion du pancréas exocrine.

Par ailleurs, l'absence totale d'un effet de la réintroduction de suc pancréatique, soit au niveau du jejunum, soit à celui de l'iléon, souligne l'importance du duodénum dans le mécanisme impliqué.

Enfin, les réponses du pancréas aux perfusions et injections intraveineuses de sécrétine et de glucagon peuvent être considérées comme des arguments en faveur d'un mécanisme de cette rétroaction se situant au niveau de la muqueuse duodénale et non de la cellule pancréatique.

\section{IN'TRODUC'TION}

On admet classiquement aujourd'hui que la sécrétion du pancréas exocrine se trouve sous le contrôle de mécanismes nerveux et hormonaux (THomas, I967; HARPER, 1972). Cependant et malgré le nombre important des recherches effectuées 
tant chez le Chien (Meyer et al., I970; Way et Grossman, I97I) que chez le Chat (HARPER et al., I940), l'Homme (Dreiring et Janowitz, ig60) ou le Porc (Hickson, I970 $a, b)$, on ne peut préciser l'importance relative de chacun de ces mécanismes dans la régulation de la sécrétion pancréatique.

Avant la mise en évidence de la cholecystokinine-pancréozymine (HARPER et RAPER, I943) prévalait la thèse d'une sécrétion pancréatique exocrine sous la dépendance presque exclusive d'un contrôle nerveux ('Thomas, I950). Par la suite, on n'accorda qu'un rôle minime à cette régulation au profit d'un contrôle hormonal : sels et partie fluide sous contrôle de la sécrétine, enzymes sous contrôle de la pancréozymine (Dupré, I970). Il est certain que pas un seul des résultats obtenus jusqu'à présent n'autorise un tel classement des mécanismes considérés. Rien ne permet de souligner dans quelle mesure les facteurs nerveux participent à la régulation de la sécrétion pancréatique et comment les différents mécanismes interfèrent entre eux : hormones entre elles et facteurs cholinergiques avec les hormones.

Très récemment, il a été proposé un type particulier de régulation qui fait certainement intervenir les contrôles nerveux et hormonal et dont l'étude devrait conduire à mieux définir ce problème de la régulation de la sécrétion pancréatique exocrine.

LAPORTE (I970) et LAPORTE et TRÉMOIIÈres (I973) mettent en évidence certains faits expérimentaux - diminution de la sécrétion pancréatique du Rat après injection intraduodénale de trypsine ou de pancréas - qui les amènent à émettre l'hypothèse d'une rétroinhibition de la sécrétion du pancréas par les enzymes excrétées par cet organe dans 1'intestin. Cette hypothèse est confirmée dans les expériences de GreEn et LyMan (I972).

Le but de ce travail a été de vérifier si ce phénomène existe également chez le Porc et d'essayer d'aborder l'étude de ses mécanismes.

\section{MÉTHODOLOGIE:}

$$
\text { I. - Animanx }
$$

Quatorze porcs de race Large-White, de poids vif moyen de $45 \mathrm{~kg}$ ont été utilisés. Dix porcs ont été munis d'une fistule pancréatique et d'une fistule duodénale, selon la technique décrite dans un travail précédent (CoRRING et al., I972), et sur 4 d'entre eux un cathéter a été placé en permanence dans une veine jugulaire (RÉRAT, I973).

Quatre animaux ont été équipés, en plus des fistules pancréatique et duodénale, d'un troisième cathéter placé soit dans la partie distale du jejunum, soit dans la partie distale de l'iléon $\left(5^{\circ} \mathrm{cm}\right.$ environ de la valvule iléo-caecale). La technique de mise en place de ce dernier cathéter est identique à celle utilisée pour la fistule duodénale. gicale.

L'expérimentation pour tous les animaux a débuté 8 à ro jours après l'intervention chirur-

Les porcs ont reçu un régime semi-synthétique de croissance à 16 p. Ioo de protéines distribué à raison de $I$ repas par jour, à $I 7$ heures.

La croissance, déterminée par pesée avant la mise en place des cathéters et après expérimentation, nous a montré un développement sub-normal des animaux (ro à I5 kg en un mois).

\section{2. - Collecte et réintroduction du suc pancréatique}

Le suc pancréatique était recueilli en permanence dans un flacon maintenu dans de la glace. Un échantillon de $20 \mathrm{ml}$ en moyenne était prélevé par heure ou par $\mathbf{1} / 4$ d'heure selon le cas, après lecture du volume de suc recueilli. Les dosages des protéines totales et des activités cnzymatiques ont été effectués à partir des échantillons. 
Le suc pancréatique a été réintroduit au niveau intestinal à l'aide d'une pompe péristaltique à débit variable (L. K. B. Varioperpex). Ce débit a été fixé à roo $\mathrm{ml} / \mathrm{heure}$ pour l'ensemble de nos essais.

Avant leur réintroduction, le suc pancréatique ou autres solutions perfusées ont été amenés à Ia température de l'animal, soit $38^{\circ} \mathrm{C}$.

Les heures de début de réintroduction et les durées de réintroduction ont été constamment modifiées d'une journée à l'autre, pour un même animal, de façon à éviter tout artefact dû à une évolution particulière de la sécrétion pancréatique.

\section{3. - Introduction intraduodénale de solutions autres que le suc pancréatique}

Une solution de trypsine bovine ( $\alpha 2 \times$ cristallisée) dans de l'HCl o, o I $\mathrm{N}$ (pH 3,5), une solution de sels $\mathrm{NaHCO}_{3}+\mathrm{NaCl}$ et du suc pancréatique déprotéinisé ont été injectés dans le duodénum à l'aide de la pompe péristaltique au débit horaire de $100 \mathrm{ml}$.

La solution de trypsine a été préparée de façon à obtenir une concentration en enzyme identique à celle du suc pancréatique et déterminée par dosage de l'activité dans ce dernier, avec de la trypsine bovine comme standard.

La composition de la solution saline est identique à celle déterminée dans un suc pancréatique de porc (sodium I6o méq./l; $\mathrm{HCO}_{3} 35$ méq./1).

Pour obtenir le suc pancréatique déprotéinisé, nous avons réalisé le schéma suivant : précipitation des protéines par addition au suc pancréatique de 8 volumes d'éthanol à 82 p. Ioo; filtration; évaporation sous vide de l'éthanol. Une mesure des activités enzymatiques nous a permis de constater l'élimination totale des enzymes et nous avons observé une très légère augmentation du $\mathrm{pH}(8,4$ à 8,8$)$.

\section{4. - Perfusions intraveineuses}

Une solution de sécrétine ( $\left.{ }^{1}\right)$ dans du sérum physiologique stérile a été perfusée par le cathéter placé dans une jugulaire de l'animal à raison de $\mathrm{I}$ unité/kg/h et à un débit de $74 \mathrm{ml} / \mathrm{heure}$.

De plus, nous avons injecté une solution de Glucagon $\left(^{2}\right)$ à une dose de $30 \mu \mathrm{g} / \mathrm{kg}$.

Sur le suc pancréatique recueilli ont été déterminées :

les protéines totales selon la méthode de Lowny (I95I) avec comme protéine de référence l'albumine porcine fraction $\mathrm{V}$.

les activités enzymatiques de la trypsine et de la chymotrypsine après activation des pro. enzymes, par la mesure titrimétrique de l'hydrolyse de substrats synthétiques (respectivement BAEE et ATEE), l'activité enzymatique de la lipase par mesure de l'hydrolyse d'une huile d'olive et de l'amylase après dégradation d'un amidon.

Les techniques de détermination des activités enzymatiques sont décrites dans un travail précédent (CorRing et al,, I970).

\section{RÉSULTATS}

\section{Réintroduction du suc pancréatique au niveau du duodénum}

Un exemple de l'évolution de la sécrétion pancréatique en réponse aux opérations de réintroduction et d'arrêt de réintroduction du suc pancréatique est reporté sur la figure $I$. Sur un porc à jeun depuis $I_{5}$ heures au minimum et soumis à une réintroduction de suc pancréatique, la sécrétion exocrine du pancréas peut être qualifiée de basale. L'arrêt de la réintroduction entraîne dans l'heure qui suit une augmentation de la quantité de suc recueilli, tant pour le volume que pour les - protéines totales. Le volume est augmenté en moyenne 5 fois et les protéines $I, 5$ à 2 fois. Cet accroissement se maintient 3 à $4 \mathrm{~h}$ puis son importance diminue lorsque le suc pancréatique n'est pas retourné à l'animal.

(1) G. I. H. Stockholm lot no I7 $35 \mathrm{I}$.

(2) NOVO lot ED 3I9. 


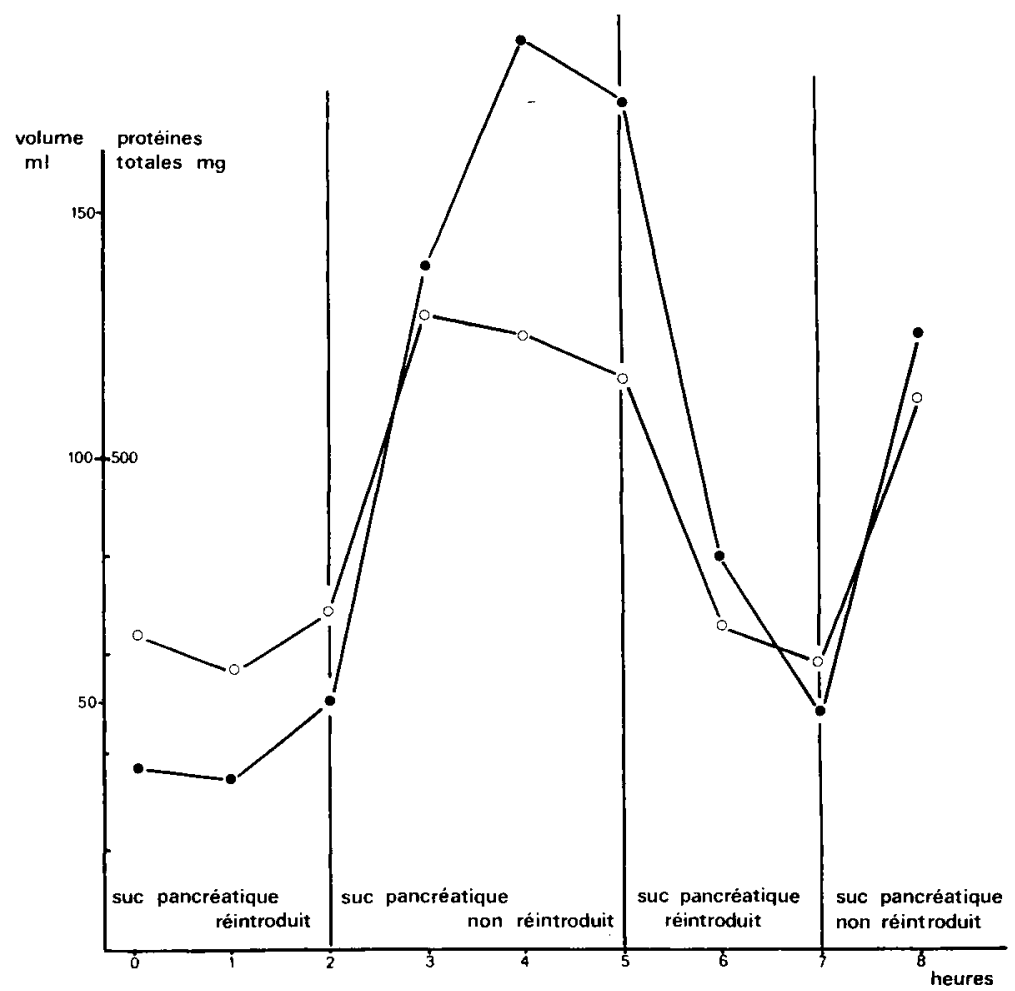

FIG. I. - Sécrétion exocrine du pancréas avec et sans réintroduction de suc pancréatique dans le duodénum, chez le Porc

$\bigcirc \longrightarrow$ protéines totales;

$\bullet$ volume.

TABLEAU I

Évolution des données mesurées dans l'étude de la sécrétion pancréatique du Porc sous l'effet de la rétroaction négative enzymatique

(Porc no 2 I I9260; journée du 5-oI-73)

\begin{tabular}{|c|c|c|c|c|c|c|}
\hline & \multicolumn{2}{|c|}{$\begin{array}{l}\text { Suc pancréatique } \\
\text { réintroduit }\end{array}$} & \multicolumn{2}{|c|}{$\begin{array}{l}\text { Suc pancréatique } \\
\text { non réintroduit }\end{array}$} & \multicolumn{2}{|c|}{$\begin{array}{l}\text { Suc pancréatique } \\
\text { réintroduit }\end{array}$} \\
\hline & 1re h & $2 \mathrm{e} \mathrm{h}$ & $3^{\text {e }} \mathrm{h}$ & $4^{\mathrm{e}} \mathrm{h}$ & 5 e $h$ & $6^{\mathrm{e}} \mathrm{h}$ \\
\hline Volume (ml) & 66 & 25 & 225 & $2 x^{\prime} 0$ & 104 & 43 \\
\hline Protéines totales $(\mathrm{mg}) \ldots \ldots \ldots$ & 400 & 620 & 1125 & 1190 & 520 & 155 \\
\hline Chymotrypsine totale (1) ..... & 10150 & 12350 & 28800 & 30000 & 12000 & 4000 \\
\hline Trypsine totale $\left({ }^{2}\right) \ldots \ldots$ & 1580 & 2500 & 4050 & 4320 & 2080 & 690 \\
\hline Lipase totale $\left({ }^{3}\right)$ & 16830 & 22000 & 36000 & 48000 & 18000 & 6300 \\
\hline Amylase totale $10^{3}\left({ }^{4}\right) \ldots \ldots$ & 1480 & 2035 & 3800 & 4640 & 2115 & 720 \\
\hline
\end{tabular}

(1) Micromoles ATEE hydrolysé, par minute.

(2) Micromoles BAEE hydrolysé, par minute.

$\left({ }^{3}\right)$ Micromoles acides gras libérés par minute.

(4) Unités amylase $10^{3}$. 
Cependant, lorsque la réintroduction du suc pancréatique est reprise 2 à 3 heures après l'accroissement observé, le volume et les protéines totales diminuent jusqu'à des valeurs en général identiques à celles enregistrées pour une sécrétion basale. Par ailleurs, le tableau I montre que les activités enzymatiques varient dans le même sens que les protéines totales et le volume.

L'exploitation statistique classique (analyse de variance) des données brutes obtenues sur les I4 animaux s'avère impossible à effectuer étant donné les fortes variations individuelles aussi bien dans le niveau de la sécrétion mesurée que dans la chronologie des variations.

C'est la raison pour laquelle nous avons eu recours à l'analyse factorielle des correspondances (TOMASSONE, I970). Cette analyse permet de calculer des axes d'inertie, d'une part à partir de la matrice des distances entre toutes les observations prises 2 à 2 dans un espace ayant pour dimensions le nombre de variables, et d'autre part, à partir de la matrice des distances entre ces mêmes variables dans un espace ayant pour dimensions le nombre d'observations. La projection sur ces axes d'inertie (orthogonaux dans un espace à $n$ dimensions) de chaque point représentatif des 2 ensembles observations et variables, permet de caractériser chaque observation par la ou les variables les plus proches, et inversement.

Ainsi l'exploitation de l'ensemble des données obtenues sur une série de réintroductions et d'arrêts de réintroduction de suc pancréatique dans une même journée,

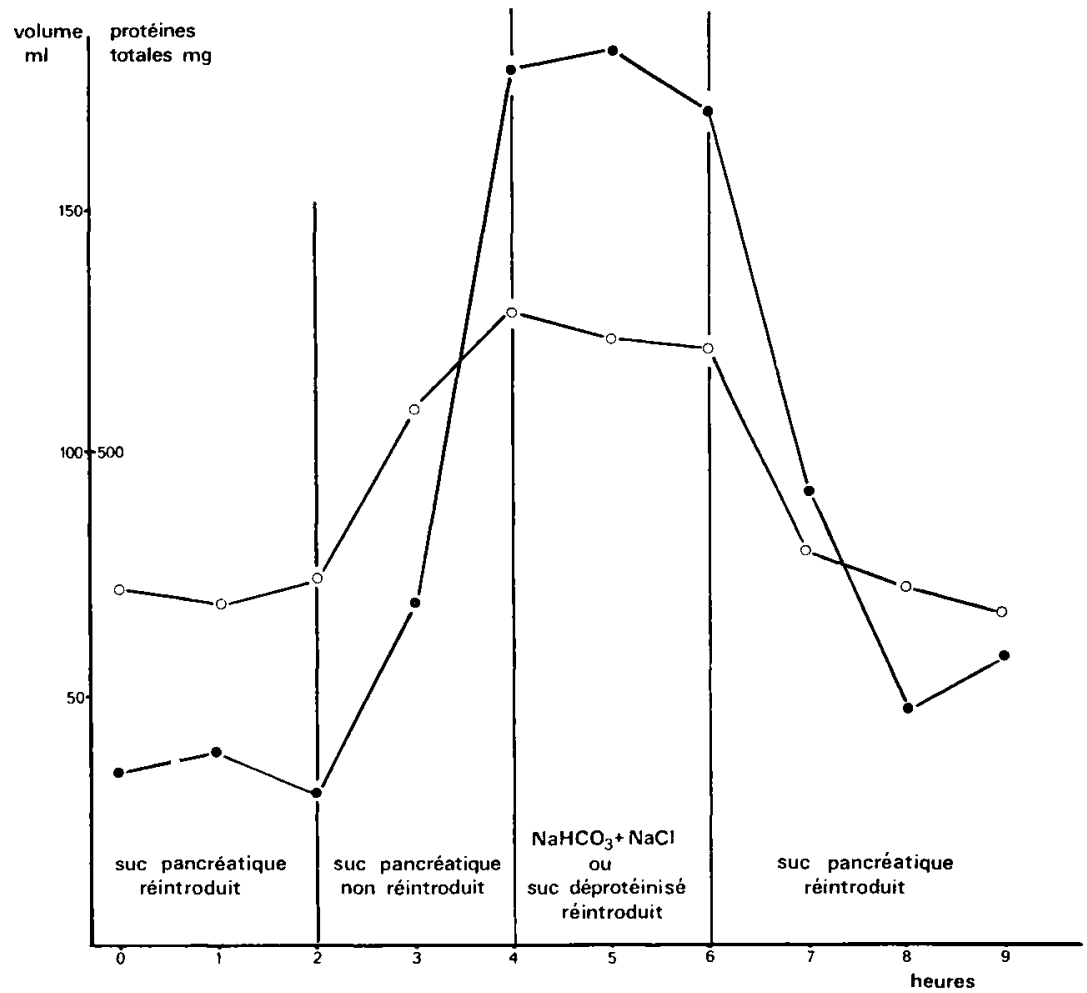

FIG. 2. - Effet de la perfusion intraduodénale d'une solution de sels $\left(\mathrm{NaHCO}_{3}+\mathrm{NaCl} ; \mathrm{pH} 8,4\right)$ ou d'un suc pancréatique déprotéinisé ( $\mathrm{pH} 8,8$ ), sur la sécrétion pancréatique exocrine du Porc - - p protéines totales; - volume. 
confirme l'effet décrit de ces opérations sur la sécrétion du pancréas et montre une évolution comparable du volume et des protéines. Elle indique enfin que $55 \mathrm{p}$. Ioo de la variabilité des données peuvent être expliqués spécifiquement par l'alternance des opérations de réintroduction et d'arrêt de réintroduction de suc pancréatique.

\section{Introduction intraduodénale de solutions salines}

Si l'on remplace le suc pancréatique réintroduit soit par une solution de sels $\left(\mathrm{NaHCO}_{3}+\mathrm{NaCl}\right)$ dont on a fait varier la quantité jusqu'à introduire 4 à 5 fois celle normalement obtenue dans un suc pancréatique stimulé, soit par un suc pancréatique déprotéinisé (fig. 2), on constate que ces introductions n'ont aucun effet inhibiteur sur la sécrétion pancréatique.

\section{Introduction intraduodénale d'une solution de trypsine}

La figure 3 montre qu'une solution de trypsine $(I, 5 \mathrm{mg} / \mathrm{ml})$ introduite au niveau du duodénum (IOO $\mathrm{ml} / \mathrm{h}$ ), après exclusion de la sécrétion, entraîne une chute de cette dernière identique à celle enregistrée lorsque le suc pancréatique est réintroduit.

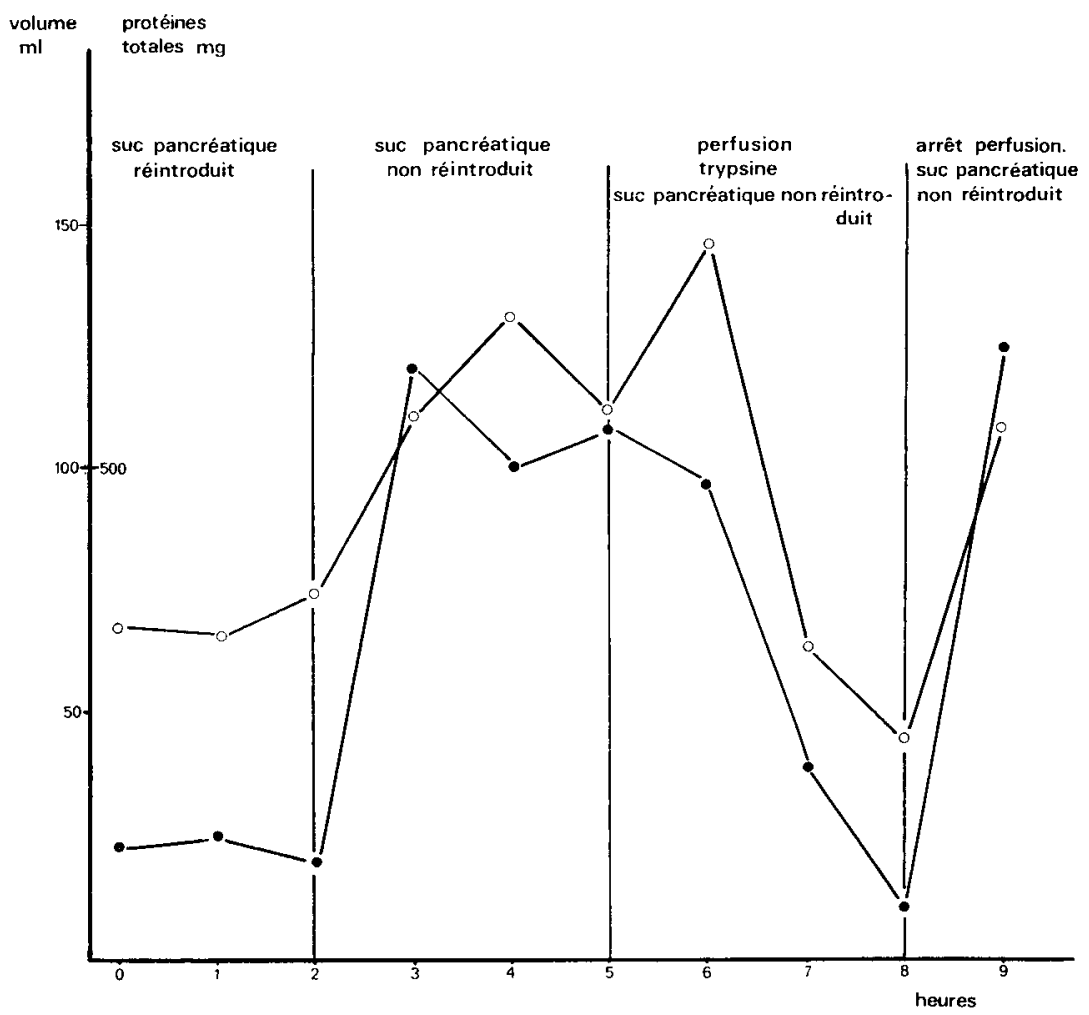

FIG. 3. - Effet de la perfusion intraduodénale d'une solution de trypsine (I,5 $\mathrm{mg} / \mathrm{ml}, \mathrm{pH} 3.5$ ? sur la sécrétion pancréatique exocrine du Porc

- - protéines totales ;

volume 
Et cela malgré le $\mathrm{pH}$ acide de la solution trypsique. Iorsque la perfusion est arrêtée, on observe une augmentation de la quantité de suc recueilli aussi bien en volume qu'en protéines totales.

\section{Réintroduction de suc pancréatique dans le jejunum et l'iléon}

Sur un même animal, porteur d'une fistule duodénale et d'une fistule jéjunale ou iléale, la réintroduction de suc pancréatique à ces dəux derniers niveaux n'a aucun effet sur la sécrétion pancréatique stimulée par une non-réintroduction de suc. Lorsque le suc pancréatique est réintroduit, au niveau duodénal, dans les heures qui suivent et chez le même animal, l'inhibition de la sécrétion apparaît aussitôt.

\section{Perfusion intraveineuse de sécrétine \\ pendant la réintroduction de suc pancréatique au niveau duodénal}

Sur un porc à jeun dont le suc pancréatique est réintroduit en permanence depuis plus de $\mathrm{I} 8 \mathrm{~h}$, nous avons effectué une perfusion intraveineuse pendant 3o minutes (débit $74 \mathrm{ml} / \mathrm{h}$ ) de sécrétine ( $\mathrm{I} \mathbf{u} / \mathrm{kg} / \mathrm{h}$ ). Nous constatons (fig. 4) que la sécrétion volumique est très fortement stimulée durant la perfusion, malgré la réintroduction permanente du suc. Cette expérience répétée plus de 5 fois sur des porcs différents conduit au même résultat.

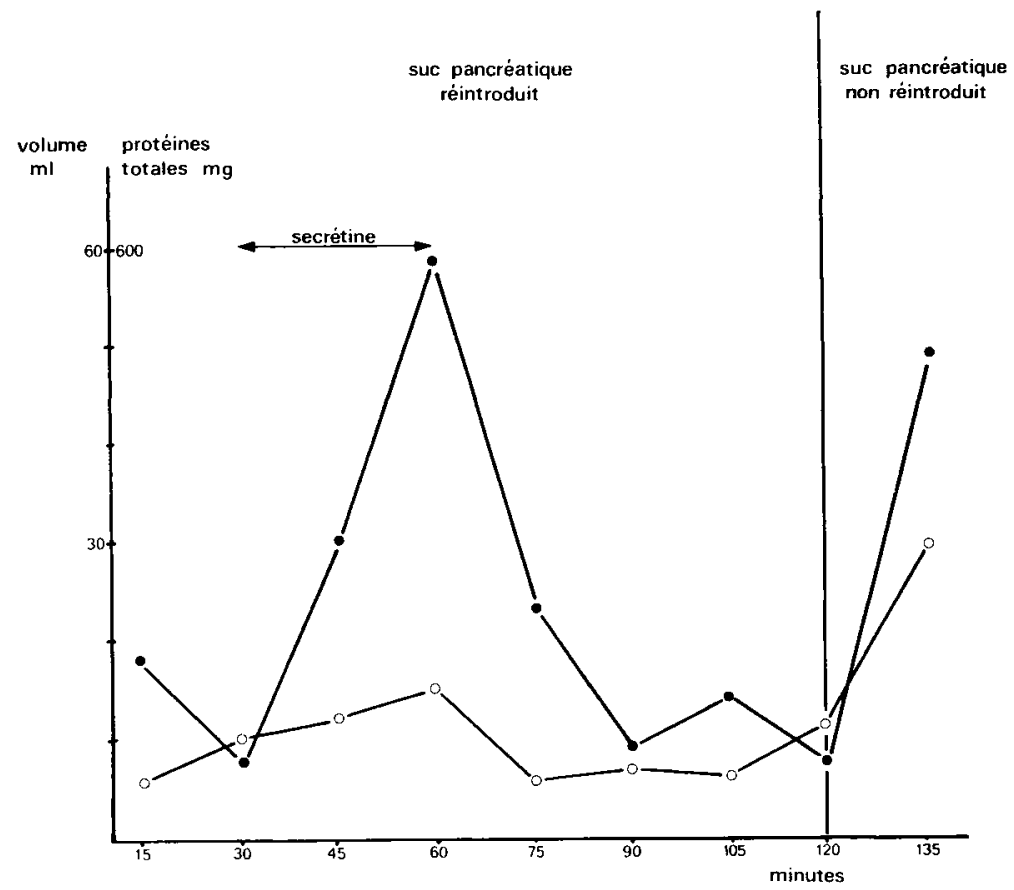

FıG. 4. - Effet de la perfusion intraveineuse d'une solution de sécrétine ( $\mathrm{I} \mathrm{u} / \mathrm{kg} / \mathrm{h}$, débit $74 \mathrm{ml} / \mathrm{h}$ ) sur la sécrétion pancréatique exocrine du Porc avec réintroduction de suc pancréatique dans le duodénum

- o protéines totales 


\section{Injection intraveineuse de glucagon}

pendant l'augmentation de la sécrétion due à l'absence de réintroduction du suc pancréatique

Après avoir observé 1'augmentation de la sécrétion pancréatique sur un porc dont la réintroduction de suc, assurée pendant plus de $\mathrm{I} 8 \mathrm{~h}$, est arrêtée, nous avons effectué une injection de glucagon au niveau de la jugulaire à une dose de $30 \mu \mathrm{g} / \mathrm{kg}$. La figure 5 montre que cette injection a comme effet une profonde inhibition de la sécrétion pancréatique et cela malgré l'arrêt de la réintroduction. Le volume et la quantité de protéines totales sont diminués sous l'effet du glucagon.

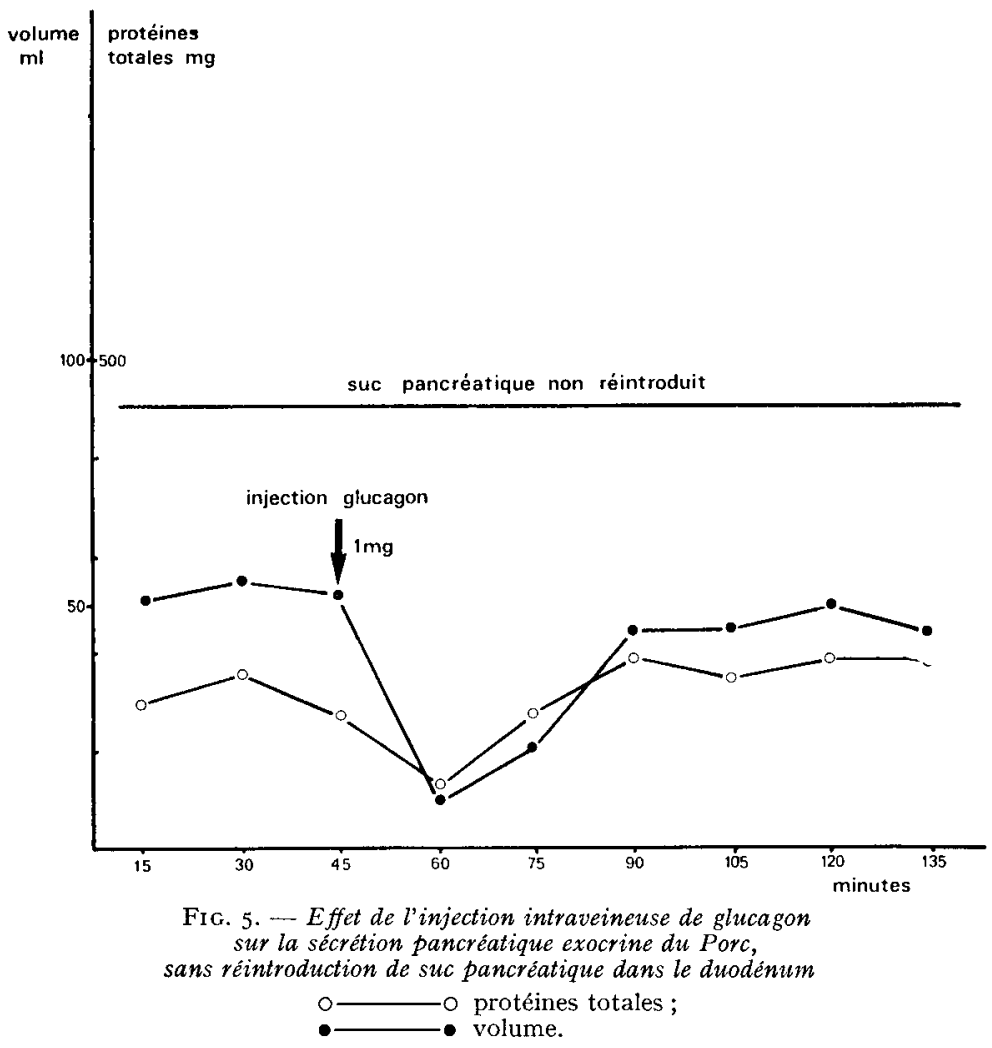

\section{DISCUSSION}

Les résultats obtenus soulignent en premier lieu un effet du suc pancréatique sur la sécrétion du pancréas exocrine du porc, effet identique à celui décrit par GREEN et Lymax (I972) chez le Rat. L'exclusion du suc pancréatique hors de l'animal entraîne un accroissement de la sécrétion tandis que sa réintroduction au niveau duodénal conduit à une inhibition des valeurs enregistrées. Cet effet fondamental n'a pas été observé par LAPORTE (I970) et LAPORTE et TrḱMOLIÈrEs (I973). Ces auteurs n'ont effectué aucune réintroduction de suc pancréatique et se sont 
limités à l'utilisation de trypsine et de ses inhibiteurs ainsi que de pancréas pour étayer leur hypothèse.

L'augmentation de la sécrétion pancréatique ne peut être en aucun cas due à l'effet classiquement décrit du $\mathrm{pH}$ acide sur la libération de sécrétine. En effet, la perfusion intraduodénale d'une solution de $\mathrm{NaHCO}_{3}+\mathrm{NaCl}(\mathrm{pH} 8,4)$ même en quantité 3 à 4 fois supérieure à celle trouvée dans un suc pancréatique, ou d'un suc pancréatique dont les protéines ont été éliminées n'a aucune action inhibitrice sur une sécrétion stimulée. Ce résultat identique à celui de GREEN et LYMAN (I972) est cependant différent de celui d'ANNIS et HALLENBECK (I95I) qui avaient observé une diminution de la sécrétion pancréatique sous l'effet d'une perfusion intraduodénale de bicarbonate alors que le suc pancréatique était dérivé à l'extérieur de l'animal. Néanmoins, la perfusion au niveau duodénal d'une solution de trypsine dissoute dans $\mathrm{HCl}$ o, oor $\mathrm{N}$, dans ce travail confirme 1'absence d'un effet $\mathrm{pH}$ et souligne également que seule la partie enzymatique du suc pancréatique, dont les enzymes protéolytiques, est responsable du phénomène de rétroaction négative observé.

Si l'on examine plus en détails la réponse du pancréas à la présence ou l'absence du suc pancréatique ou de trypsine dans le duodénum, on constate que le volume et la quantité de protéines totales sont sensibles à la rétroaction négative. Cela laisserait supposer un mécanisme à double effet : d'une part une stimulation du volume de suc excrété, d'autre part une stimulation de la sécrétion des protéines enzymatiques. LAPORTE (I970) ne mentionne d'abord que le seul effet important observé, celui sur l'accroissement des protéines enzymatiques bien qu'ayant étudié les deux effets. Par la suite LAPORTE et TRÉMOLIÈRES (I973), reprenant les résultats obtenus par LAPORTE (I970) et les ayant soumis à une analyse plus fine, montrent une petite diminution très significative du débit hydrique après injection de pancréas, trypsine, pancréas et trypsine. De leur côté, GREEN et LYMAN (I972) démontrent une forte augmentation de la sécrétion enzymatique en 1'absence de réintroduction de suc pancréatique et observent un léger, mais non-proportionnel, accroissement du volume recueilli. Dans le cas du Porc, les variations de volume, très importantes (de l'ordre de 500 p. IOo), s'accompagnent de modifications moindres de la quantité d'enzymes (de l'ordre de 150 à $200 \mathrm{p}$. I00), l'augmentation du volume pourrait entraîner une excrétion accrue des enzymes (" washing-out »). Cependant, cela semble peu probable puisque par exemple l'injection ou la perfusion intraveineuse de sécrétine conduit à un lessivage de la glande en moins d'une heure, alors que dans le cas présent l'augmentation des protéines totales se poursuit durant 2 à 3 heures lorsque 1'on maintient cet accroissement de la sécrétion par non-réintroduction du suc pancréatique. Ce n'est que vers la $4^{\mathrm{e}}$ ou $5^{\mathrm{e}}$ heure que l'on assiste à une diminution progressive parallèle du volume et des enzymes, due vraisemblablement à un épuisement de la glande.

L es expériences de réintroduction de suc pancréatique, soit dans le jejunum, soit dans l'iléon, montrent par comparaison avec la réintroduction du même suc au niveau duodénal, que le duodénum semble jouer un rôle essentiel dans la régulation de la sécrétion par rétroaction négative. Un rôle analogue de cette partie postpylorique de l'intestin a été mis en évidence par ANDERson et al. (1965, I970). Ces auteurs ont suggéré que la première partie du duodénum est un facteur essentiel dans l'inhibition d'acide gastrique par l'acide dans l'intestin. Par ailleurs, KonTUREK et al. (I972) ont montré que la quantité de pancréozymine libérée, maximum 
dans le duodénum, décroît dans les parties distales de l'intestin et n'est plus décelable dans l'iléon par la méthode utilisée. Une étude identique concernant la sécrétine montre que l'activité pancréatique en réponse à la sécrétine stimulée par l'injection d'HCl dans différentes parties de l'intestin diminue en fonction de la distance du pylore et n'est plus dans l'iléon que de io p. Ioo de celle trouvée dans le duodénum (KoNTUREK et al., I97I).

Enfin, GREEN (I97I) a montré que la trypsine inactivée n'induit au niveau duodénal aucune inhibition de la sécrétion pancréatique du Rat et ce contrairement à la trypsine active. Nous pouvons supposer au vu de ces derniers résultats, que la rétroaction négative a tout d'abord comme base d'action principale le duodénum. Par ailleurs, étant donné le double effet observé sur le volume et la quantité de protéines totales et les résultats de KONTUREK et de GREEN, l'action de ce phénomène se traduirait par un effet des enzymes pancréatiques au niveau des cellules sécrétagogues des hormones sécrétine et pancréozymine, en inhibant leur synthèse ou leur libération.

Ce travail n'a pas la prétention de vérifier une telle hypothèse ; cependant la perfusion de sécrétine et l'injection de glucagon peuvent être considérées comme arguments en sa faveur. En effet, nous avons vu que la sécrétine perfusée par voie intraveineuse $(\mathrm{IU} / \mathrm{kg} / \mathrm{h})$ stimule la sécrétion pancréatique bien que l'animal soit soumis à une réintroduction permanente de suc. De même DYck et al. ont montré chez le Chien (1969) et chez l'Homme (1970) que le glucagon inhibe la sécrétion du pancréas par compétition avec la sécrétine au niveau de la cellule pancréatique. Les essais réalisés sur le Porc dont le suc pancréatique n'est pas réintroduit conduisent également à une diminution accrue de la sécrétion. Ce qui laisserait supposer que l'hyperstimulation de la sécrétion en période de non-réintroduction est due à un effet de la sécrétine endogène. La diminution des protéines totales laissent également envisager une compétition sélective au niveau de la cellule pancréatique, du glucagon et de la pancréozymine.

En conclusion, le phénomène de rétroaction négative par les enzymes du pancréas semble être un type de régulation de la sécrétion pancréatique du Porc. Le mécanisme de cette régulation se situerait au niveau du duodénum sous l'effet des enzymes protéolytiques qui inhiberaient la synthèse ou la libération des hormones sécrétine et pancréozymine. Des travaux en cours ont pour but de vérifier une telle hypothèse qui par ailleurs s'accorde parfaitement à celle émise par GrEFN et LYMAN (I973), mais s'oppose à la théorie de LAPORTE et TRÉmOLIÈRES (I973) - passage de la trypsine dans le sang et inhibition de la trypsine dans le sang par la pancréozymine qui repose sur une argumentation expérimentale nettement insuffisante et certainement criticable.

Reçu pour publication en février 1974.

\section{SUMMARY}

FEED-BACK REGUIATION OF PANCREATIC SECRE'TION IN THE PIG

It is classically admitted that regulation of the secretion from the exocrine pancreas is controlled by nervous and hormonal mechanisms. However, the role cannot be defined yet with accuracy. 
Some research work on the rat (LAPORTE and TRÉMOLIÈRES, I973; GREEN and LYMAN, 1972) permits to suppose the existence of a type of regulation through a feed-back mechanism. by enzymes excreted by the pancreas and released into the intestine.

The aim of the present work is to verify and study this phenomenon in the pig.

In pigs where a permanent reintroduction of pancreatic juice was made at the level of the duodenum, ceasing this operation led within one hour to an increase in the amount of total proteins $\left(X_{1} \mathrm{I}_{-5}-2\right)$ and the volume of juice collected $\left(X_{5}\right)$. As soon as the pancreatic juice was reintroduced into the animal, the secretion recovered its minimal value. A mixture of salts $\left(\mathrm{NaHCO}_{3}+\mathrm{NaCl}\right)$, representative of those in the pancreatic juice, or a deproteinized pancreatic juice did not have any inhibitory effect on the secretion of the pancreas when perfused into the duodenum.

Furthermore, intraduodenal perfusion of a solution of active trypsin, even at acid $\mathrm{pH}$, resulted in a lowering of pancreatic secretion identical to that induced by the reintroduced pancreatic juice.

Consequently, it seems that only enzymes, and proteolytic ones, induce this phenomenon of feed-back control of exocrine pancreatic secretion. In addition, the total absence of any effect when reintroducing pancreatic juice, either at the level of the jejunum or the ileum, emphasizes the role of the duodenum for the mechanism involved.

Finally, the responses of the pancreas to perfusions or intravenous injections of secretine and glucagon indicate that this mechanism acts at the level of the duodenal mucosa and not in the pancreatic cell.

\section{RÉFÉRENCES BIBLIOGRAPHIQUES}

Anderson S., Nilsson G., Uvnäs B., I965. Inhibition of gastric secretion by acid in proximal and distal duodenal pouches. Acta Physiol. Scand., 65, 191-192.

Anderson S., Nilsson G., Sjodin L., UvNäs B., I97o. Mechanism of duodenal inhibition of gastric acid secretion. In : Nobel Symp. XVI : Frontiers in Gastrointestinal Hormone Research, Stockholm, 1970.

Annis D., Hallenbeck G. A., I95I. Effect of excluding pancreatic juice from duodenum on secretory response of pancreas to a meal. Proc. Soc. Exp. Biol. and Med., y7, 383-385.

Corring T., Aumaitre A., I970. Effet du traitement thermique du tourteau de soja sur la variation de l'équipement enzymatique du pancréas exocrine et les performances pondérales du Rat aptès sevrage. Ann. Biol. anim. Bioch. Biophys., 10, 443-457.

Corring T., Aumaitre A., Rerat A., 1972. Fistulation permanente du pancréas exocrine chez le Porc. Application : réponse de la sécrétion pancréatique au repas, Ann. Biol. anim. Bioch. Biophys., 12, $109-124$.

Dreiling D. A., Janowitz H. D., I960. Effect of some anticholinergie drugs on basal and secretionstimulated pancreatic secretion in man. Federation Proc., 19, 189.

DUPre J., 197o. Regulation of the secretions of the pancreas. Ann. Rev. Med., 21, 299-3I6.

Dyck W. P., Rudick J., Hoexter B., Janowitz H. D., ig69. Influence of Glucagon on pancreatic exocrine secretion. Gastroenterology, 56, $53 \mathrm{I}-537$.

Dyck W.P., Texter E. C., Lasater J. M., Hightower N. C,, ig7o. Influence of glucagon on pancreatic secretion in man. Gastroenterology, 58, 532-539.

Green G. M., I97I. Ph D Thesis, Dept. of Nutr. Sci. Univ. of California. Berkeley, C A.

GreEN G. M., LymaN R. L., I972. Feed back regulation of pancreatic enzyme secretion as a mechanism for trypsin Inhibitor-Induced Hypersecretion in Rats. Proc. Soc. Exp. Biol. and Med., 140, 6-I 2.

Green G. M., Olds Barbara A., Matthews Glenda, Lyman R. L., I973. Protein, as a regulator of pancreatic enzyme secretion in the Rat. Proc. Soc. Exp. Biol. Med., 142, II62-II67.

Harper A. A., Raper H. S., I943. Pancreozymin, a stimulant of the secretion of pancreatic enzyme in extracts of the small intestine. J. Physiol., London, 102, II5-125.

Harper A. A., VAss C. C. N., r94r. The control of the external secretion of the pancreas in cats. $J$. Physiol., London, 99, 4I5-435.

Harper A. A., r972. Progress report. The control of pancreatic secretion. Gut., 13, 308-317.

Hickson J. C. D., I97o $a$. The secretory and vascular response to nervous and hormonal stimulation in the pancreas of the Pig. J. Physiol., 206, 299-322.

Hickson J.C. D., I97o $b$. The secretion of pancreatic juice in response to stimulation of the vagus nerves in the Pig. J. Physiol., 206, 275-297.

Konturek S. J., TAsler J., OBtulowicz W., r97I. Localization of secretion release by acid in small intestine of the Dog. Am. J. Physiol., 220, 124-127.

Konturek S. J., Tasier J., Obtulowicz W., I972. Localization of cholecystokinin release in intestine of the Dog. Am. J. Physiol., 22, 16-20. 
Laporte J. C., I970. Activation et sécrétion des enzymes pancréatiques. Thèse sciences, Paris.

LAPORTE J. C., TRÉmolières J., I973. Action de la trypsine et des inhibiteurs trypsiques sur la sécrétion pancréatique. Nutr. Métabol., 15, 192-206.

Meyer J. H., Way L. W., Grossman M. I., I97o. Pancreatic response to acidification of various lengths of proximal intestine in the Dog. Am.J. Physiol., 219, 97I-977.

Rerat A., 1973. Non-publié.

Thomas J. E., 1950. The external secretion of the pancreas. Springfield Ill : Thomas.

Tномаs J. E., I967. Neural regulation of pancreatic secretion. In : Handbook of Physiology. Section 6 , 2, 955-968.

Tomassone R., 197o. L'analyse factorielle des correspondances. $3^{\text {e }}$ CongrèsJ. U. F. R. O. du groupe des statistiques forestières. 7-I I, 9. I. N. R. A. Jouy en Josas, In : " No spécial »I. N. R. A., I972, I6I-I73.

Way L. W., Grossman M. I., 1971. Gastric and pancreatic response to acid in the duodenum after partial duodenectomy. Am. J. Physiol., 220, гог6-гоrg. 